\title{
Shoulder Palsy due to Middle Cerebral Artery Embolism
}

\author{
Hiromasa Tsuda ${ }^{\mathrm{a}, \mathrm{b}}$, Sayaka Nagao ${ }^{\mathrm{a}}$, Yuto Yamazaki ${ }^{\mathrm{a}}$, Kozue Tanaka ${ }^{\mathrm{a}}$
}

\begin{abstract}
An 80-year-old Japanese woman with paroxysmal atrial fibrillation suddenly developed sensory aphasia, with moderate weakness restricted to the right shoulder with normal muscle strength in the elbow, wrist and fingers. There were no other neurological abnormalities. Diffusion-weighted cranial magnetic resonance imaging demonstrated scattered infarct lesions in the territory of the left middle cerebral artery (MCA), probably due to cardiogenic embolism. Right-sided shoulder palsy might have been caused by a localized infarct lesion in the precentral gyrus on the left side, and resolved within 10 days. Because the area corresponding to the shoulder is small in the primary motor cortex, isolated shoulder palsy due to cortical infarction is very rare. Its etiology remains controversial, but borderzone ischemia between the anterior cerebral artery and MCA, and atherothrombosis of the cortical branch of MCA are proposed. Our case shows that the area corresponding to the shoulder receives blood from the cortical branch of MCA.
\end{abstract}

Keywords: Aphasia; Atrial fibrillation; Homunculus; Precentral gyrus; Primary motor cortex

\section{Introduction}

Isolated shoulder palsy (ISP) is defined as unilateral shoulder motor weakness without any other neurological deficit, which is caused by pyramidal tract disorder [1-4]. In 1937, based on electrical stimulation of the brain surface during surgery, Penfield et al [5] reported a broadly somatotopic representation of the different body parts arranged in the pri-

\footnotetext{
Manuscript accepted for publication October 25, 2012

a Department of Neurology, Tokyo Metropolitan Cancer and Infectious Diseases Center Komagome Hospital, 3-18-22, Honkomagome, Bunkyo-ku, 113-8677, Tokyo, Japan

${ }^{b}$ Corresponding author: Hiromasa Tsuda, Department of Neurology,

Tokyo Metropolitan Cancer and Infectious Diseases Center

Komagome Hospital, 3-18-22, Honkomagome, Bunkyo-ku, 113-8677,

Tokyo, Japan. Email: hiromasatsuda@cick.jp

doi: http://dx.doi.org/10.4021/jmc936w
}

mary motor cortex. Because the area corresponding to the shoulder is very small, to date there have been only 4 reported cases of ISP due to a cortical infarction, and its vascular supply remains controversial [1-4]. Here, we describe a case of shoulder palsy probably due to cardiogenic embolism, which proves that the area corresponding to the shoulder receives its blood from the cortical branch of MCA.

\section{Case Report}

An 80-year-old Japanese woman with paroxysmal atrial fibrillation suddenly presented with sensory aphasia and difficulty in lifting the right arm, despite no shoulder pain in June 2012; therefore, the patient was admitted to our hospital. Consciousness was alert. General examination demonstrated no abnormalities. Cranial nerve impairment was not detected. The patient was right-handed. Hand grasping power was 12 $\mathrm{kg}$ in the right and $10 \mathrm{~kg}$ in the left. In the upper extremities, the right-sided Barre sign was positive. In the lower extremities, neither the Barre sign nor Mingazzini sign was positive on the left side. Manual muscle testing of the left upper limb demonstrated that the pectoralis major, deltoid, supraspinatus, infraspinatus, rhomboideus, serratus anterior, and latissimus dorsi were grade 3 , although the trapezius, biceps brachii, triceps brachialis, brachioradialis, extensor carpi radialis longus, extensor carpi ulnaris, flexor carpi radialis, and flexor carpi ulnaris were grade 5. Bend, extension, and wiggle of the fingers were normal on both sides. Muscle tonus was normal in the extremities. Muscle atrophy and fasciculation were not observed. Deep tendon reflexes were all normal and pathological reflexes were not detected in the extremities. Superficial sensation (touch sensation, pain sensation, temperature sensation, and topesthesia), deep sensation (joint sensation and vibratory sense), and combined sensation (two-point discrimination, graphaesthesia, stereognosis, and double simultaneous stimulation) were all normal. The finger-nose test, nose-finger-nose test, hyperpronation test, and hand pronation supination test were all normal. Truncal ataxia was not observed. In the gait test, arm swing was slow on the left side. There were no other neurologic abnormalities. As a result, there was moderate weakness restricted to 


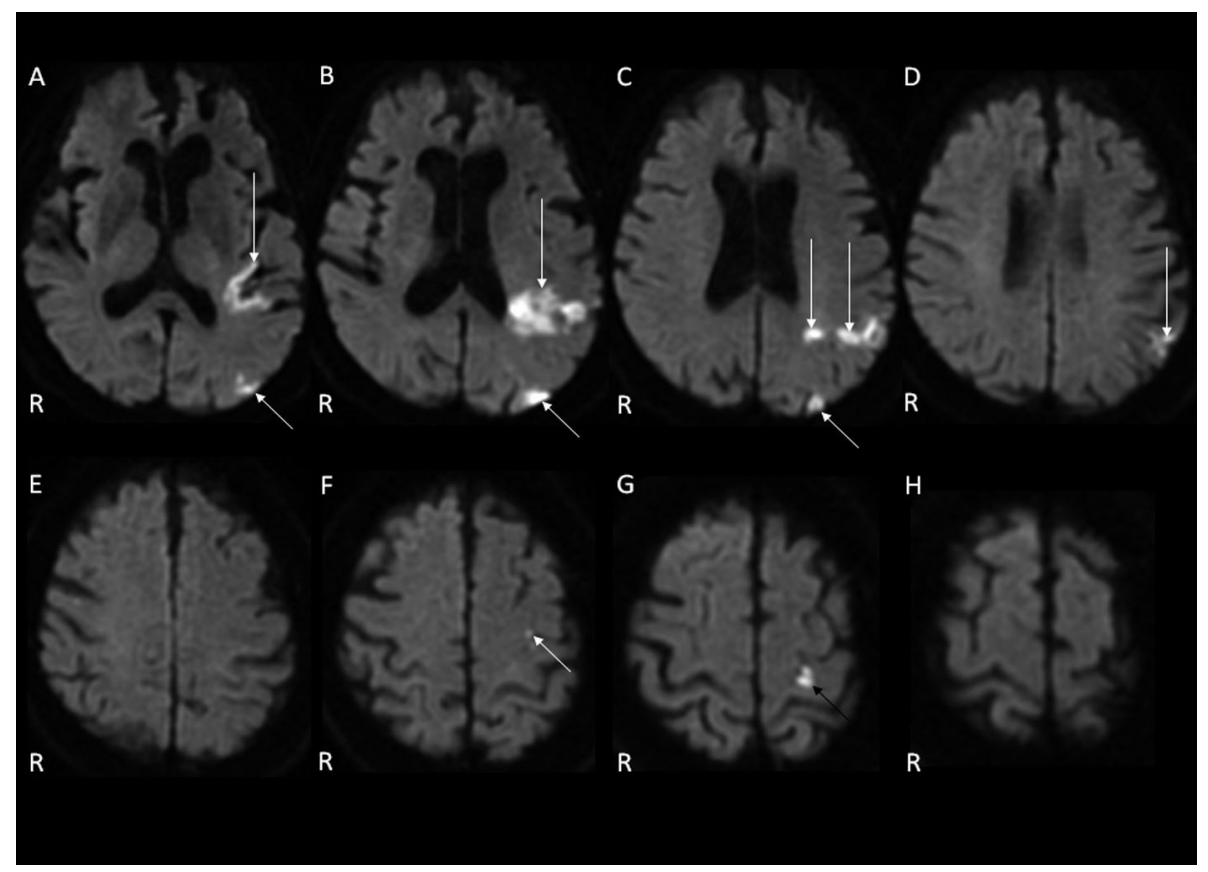

Figure 1. Cranial magnetic resonance imaging on diffusion-weighted image demonstrated scattered infarct lesions in the territory of the MCA on the left side (white arrow). In Fig. 1G, a localized infarct lesion was demonstrated in the prerencral gyrus on the left side (black arrow).

the right shoulder with normal joint flexibility, despite normal muscle strength in the elbow, wrist and fingers; therefore, the patient was diagnosed as having right-sided shoulder palsy. Complete blood cell count and blood chemistry were within normal ranges. Chest roentgenogram, electrocardiogram and echocardiogram findings were normal. Carotid ultrasonogram demonstrated normal findings. Shoulder roentgenogram demonstrated no abnormalities. Cranial magnetic resonance (MR) imaging demonstrated an infarct lesion in the territory of the MCA on the left side (Fig. 1). A localized infarct lesion was demonstrated in the precentral gyrus on the left side, which was considered responsible for the right shoulder palsy. Cranial MR angiography demonstrated normal findings. Under rehabilitation therapy, sensory aphasia resolved within 20 days and right-sided ISP within 10 days, respectively.

\section{Discussion}

Penfield et al [5] described that the motor shoulder area might be located more medially than the motor hand area in the precentral gyrus. In addition, Celebisoy et al [6] noted that the hand area in the primary motor cortex is located in the middle to lower portion of the anterior wall of the central sulcus; however, in the primary motor cortex, detailed vascular supply remains uncertain. Kim [7] speculated that the medial portion of the precentral knob representing topographically ulnar-sided fingers may correspond to the border area between the anterior cerebral artery and MCA, whereas the lateral portion representing radial-sided fingers is supplied by distal MCA branches.

In 2003, Komatsu et al [1] described the first case of ISP. Cranial diffusion-weighted MR imaging demonstrated a small infarct lesion in the precentral gyrus on the ipsilateral side to shoulder palsy. Electrocardiogram did not demonstrate the source of the embolus [1]. A borderzone infarction between the anterior cerebral artery and MCA was suspected as its pathogenesis because of severe stenosis of the internal carotid artery based on MR angiography findings; however, no MR angiography image was shown [1]. Because cerebral angiography, 3D-CTA, and carotid ultrasonogram were not performed, whether collateral circulation was present was unknown [1]. In 2006, Nah et al [2] noted a second case of ISP. Cranial MR imaging demonstrated a small infarct lesion in the right precentral gyrus [2]. Cranial MR angiography demonstrated no steno-occlusive lesions in the intracranial and extracranial arteries [2]. Electrocardiogram demonstrated atrial fibrillation and vigorous spontaneous echogenic contrast with sludge in the left atrial appendage; however, the detailed etiology of infarction was not mentioned, although intravenous heparin therapy was performed [2]. In 2007, Uncini et al [8] reported a case of unilateral shoulder palsy due to a localized cortical infarction. Analysis of functional MR imaging confirmed the location of the corresponding area to the shoulder in the precentral gyrus; however, muscle strength weakness of the biceps brachii and triceps brachialis was also observed [8]. Pathogenesis of the infarction was not mentioned [8]. In 2011, we noted a third case of 
ISP. Diffusion-weighted cranial MR imaging demonstrated a cortical infarct lesion in two serial axial images [3]. Electrocardiogram, echocardiogram, carotid ultrasonography, and cranial MR angiography demonstrated normal findings [3]. Marked blood pressure fluctuations were not observed. Consequently, atherothrombosis was suspected. After administration of an intravenous anti-platelet agent, the patient became asymptomatic within 10 days [3]. In 2012, we reported a fourth case of ISP [4]. Diffusion-weighted cranial MR imaging demonstrated infarction of the cortical branch of MCA, probably due to atherothrombosis, which involved the area corresponding to the shoulder in the primary motor cortex [4]. Therefore, we proposed that the area corresponding to the shoulder received its blood from the cortical branch of MCA. Here, in our present case, right-sided shoulder palsy was caused by an infarct lesion in the precentral gyrus on the left side, which resulted from an embolism of the MCA. Consequently, we have provided further evidence that the cortical branch of MCA may supply blood to the area corresponding to the shoulder in the precentral gyrus.

\section{Conflict of Interest}

There is no conflict of interest relevant to this article.

\section{Grant Support}

There is no grant support.

\section{References}

1. Komatsu K, Fukutake T, Hattori T. Isolated shoulder paresis caused by a small cortical infarction. Neurology. 2003;61(10):1457.

2. Nah HU, Park HK, Kang DW. Isolated Shoulder Weakness due to a Small Cortical Infarction. J Clin Neurol. 2006;2(3):209-211.

3. Tsuda H, Kubota Y, Tanaka K, Kishida S. Isolated shoulder palsy due to a cortical infarction. Intern Med. 2011;50(8):947.

4. Tsuda H, Shinozaki Y, Tanaka K, Miura Y, Kishida S, Karasawa K. Isolated shoulder palsy due to infarction of the cortical branch of the middle cerebral artery. Intern Med. 2012;51(16):2217-2219.

5. Penfield W, Boldrey E. Somatic motor and sensory representation in the cerebral cortex of man as studied by electrical stimulation. Brain 1937;69(4):389-443.

6. Celebisoy M, Ozdemirkiran T, Tokucoglu F, Kaplangi $\mathrm{DN}$, Arici S. Isolated hand palsy due to cortical infarction: localization of the motor hand area. Neurologist. 2007;13(6):376-379.

7. Kim JS. Predominant involvement of a particular group of fingers due to small, cortical infarction. Neurology. 2001;56(12):1677-1682.

8. Uncini A, Caporale CM, Caulo M, Ferretti A, Tartaro A, Ranieri F, Di Lazzaro V. Isolated shoulder palsy due to cortical infarction: localisation and electrophysiological correlates of recovery. J Neurol Neurosurg Psychiatry. 2007;78(1):100-102. 